\title{
Early Actions of Anti-Vascular Endothelial Growth Factor/Vascular Endothelial Growth Factor Receptor Drugs on Angiogenic Blood Vessels
}

\author{
Basel Sitohy, ${ }^{*}$ Sunghee Chang, ${ }^{*}$ Tracey E. Sciuto, ${ }^{*}$ Elizabeth Masse, ${ }^{*}$ Mei Shen, ${ }^{*}$ Peter M. Kang, ${ }^{\dagger}$ Shou-Ching Jaminet, ${ }^{*}$ \\ Laura E. Benjamin, ${ }^{*}$ Rupal S. Bhatt, ${ }^{\dagger}$ Ann M. Dvorak, ${ }^{*}$ Janice A. Nagy, ${ }^{*}$ and Harold F. Dvorak*
}

From The Center for Vascular Biology Research and the Department of Pathology, ${ }^{*}$ and the Department of Medicine, ${ }^{\dagger}$ Beth Israel Deaconess Medical Center, Harvard Medical School, Boston, Massachusetts

\author{
Accepted for publication \\ June 22, 2017. \\ Address correspondence to \\ Harold F. Dvorak, M.D., The \\ Center for Vascular Biology \\ Research and the Department \\ of Pathology, Beth Israel \\ Deaconess Medical Center, \\ 330 Brookline Ave., RN227C, \\ Boston, MA 02215. E-mail: \\ hdvorak@bidmc.harvard.edu.
}

\begin{abstract}
Tumors induce their heterogeneous vasculature by secreting vascular endothelial growth factor (VEGF)-A. Anti-VEGF/VEGF receptor (VEGFR) drugs treat cancer, but the underlying mechanisms remain unclear. An adenovirus expressing VEGF-A (Ad-VEGF-A ${ }^{164}$ ) replicates the tumor vasculature in mice without tumor cells. Mother vessels (MV) are the first angiogenic vessel type to form in tumors and after Ad-VEGF- ${ }^{164}$. Multiday treatments with a VEGF trap reverted MV back to normal microvessels. We now show that, within hours, a single dose of several anti-VEGF drugs collapsed MV to form glomeruloid microvascular proliferations (GMP), accompanied by only modest endothelial cell death. GMP, common in many human cancers but of uncertain origin, served as an intermediary step in MV reversion to normal microvessels. The vasodisruptive drug combretastatin CA4 also targeted MV selectively but acted differently, extensively killing MV endothelium. Antivascular changes were quantified with a novel Evans blue dye assay that measured vascular volumes. As in tumors, Ad-VEGF-A $\mathrm{A}^{164}$ strikingly increased endothelial nitric oxide synthase (eNOS) expression. The eNOS inhibitor N(G)-Nitro-L-arginine methyl ester mimicked anti-VEGF/ VEGFR drugs, rapidly collapsing MV to GMP. Inhibition of eNOS reduces synthesis of its vasodilatory product, nitric oxide, leading to arterial contraction. Patients and mice receiving anti-VEGF/VEGFR drugs develop hypertension, reflecting systemic arterial contraction. Together, anti-VEGF/VEGFR drugs act in part by inhibiting eNOS, causing vasocontraction, MV collapse to GMP, and subsequent reversion of GMP to normal microvessels, all without extensive vascular killing. (Am J Pathol 2017, 187: 2337-2347; http://dx.doi.org/10.1016/j.ajpath.2017.06.010)
\end{abstract}

The blood vessels supplying human and animal tumors are heterogeneous, of at least six distinctly different types ${ }^{1-4}$ : mother vessels (MV), glomeruloid microvascular proliferations (GMP), capillaries, stabilized MV, feeding arteries (FAs), and draining veins. This heterogeneity, and the fact that blood vessels account for only a small fraction of the tumor mass, complicate study of the tumor vasculature in situ. Therefore, because tumors generally induce the formation of new blood vessels through the vascular endothelial growth factor (VEGF)/VEGF receptor (VEGFR) pathway, ${ }^{5}$ we engineered an adenoviral vector to express murine VEGF$\mathrm{A}^{164}$ (Ad-VEGF-A ${ }^{164}$ ) and used it to produce, in sequence, surrogate forms of each type of tumor vessel in immunodeficient mice in the absence of tumor cells. ${ }^{1,6,7}$
In tumors ${ }^{8}$ and in response to Ad-VEGF-A ${ }^{164},{ }^{1,6} \mathrm{MV}$ are the first new angiogenic vessel type to form and predominate in many rapidly growing mouse tumors. ${ }^{2,8-10} \mathrm{MV}$ are

\footnotetext{
Supported by US Public Health Service NIH grants P01 CA-92644 (H.F.D.) and 5 R01 CA196996-02 (R.S.B.), by a contract from the National Foundation for Cancer Research (H.F.D.), and by the Swedish Research Council, the Medical Faculty of Umeå University, the Council of Västerbotten, and Lions Cancer Research Fund (B.S.).

Disclosures: Aflibercept and control peptide were gifts from Regeneron Pharmaceuticals (Tarrytown, NY). Combretastatin A4-phosphate was provided by Oxigene Inc. (Waltham, MA).

Current address of B.S., Department of Radiation Sciences, Umeå University, Umea, Sweden; of S.C., Department of Medicine, Weill Cornell Medical College, New York, NY; of L.E.B., KathadinRx, Inc., Bowdoinham, ME.
} 
enlarged, thin-walled, pericyte-poor, lightly fenestrated, hyperpermeable blood vessels with disrupted basement membranes; they achieve maximum numbers at about 5 days after Ad-VEGF-A ${ }^{164}$ injection. ${ }^{1,6}$ Thereafter, some MV reacquire a smooth muscle cell coat to become stabilized MV that persist indefinitely. Many other MV, however, do not stabilize and are replaced by GMP over a period of days to several weeks. ${ }^{1,6,11}$ GMP are poorly organized vascular structures with minute lumens that resemble renal glomeruli macroscopically, hence their name. ${ }^{1,6,11}$ Once formed in the Ad-VEGF-A ${ }^{164}$ setting, GMP involute over a period of weeks into normal microvessels, thereby reverting to the normal capillaries and venules from which MV originally arose in response to VEGF stimulation. ${ }^{1,3,6,11}$ The mechanisms by which GMP form and involute are poorly understood, but GMP involution has been correlated with declining local levels of VEGF because VEGF-expressing adenoviral particles are discharged stochastically from infected cells. ${ }^{6,11}$ In parallel with angiogenesis, Ad-VEGF$\mathrm{A}^{164}$ induces arteriogenesis and venogenesis as preexisting normal arteries and veins enlarge and remodel to form the FAs and draining veins that supply and drain MV.,

Antiangiogenesis is currently used to treat many types of human cancer, ${ }^{12-14}$ and nearly all antiangiogenesis drugs in current use are designed to inhibit VEGF-A or its receptors. Although highly effective against many rapidly growing mouse tumors, ${ }^{15}$ anti-VEGF/VEGFR therapy has been less successful in treating mouse tumors with an established vasculature ${ }^{16}$ and has not as yet fulfilled its promise in human cancer, even when combined with chemotherapy. ${ }^{12-14}$

A number of explanations have been offered to account for the limited effectiveness of anti-VEGF/VEGFR therapy in cancer. ${ }^{17,18}$ One possibility is that these drugs attack only a subset of tumor blood vessels, limiting their ability to cause widespread tumor damage. ${ }^{19,20}$ With the use of the Ad-VEGF-A ${ }^{164}$ model, we demonstrated that a standard course of therapy (three doses of $25 \mathrm{mg} / \mathrm{kg}$ at 3-day intervals) with aflibercept, a VEGF trap in clinical use, ${ }^{19}$ caused selective disappearance of MV, replacing them 8 days later with normal capillaries and veins, that is, the same transition that required weeks in the absence of therapy. The other angiogenic and arteriovenogenic blood vessel types induced by Ad-VEGF-A ${ }^{164}$ were apparently unaffected.

Although these studies demonstrated that aflibercept acted selectively on MV, they were not empowered to elucidate the steps or mechanisms by which aflibercept caused MV disappearance and replacement by normal microvessels. Therefore, we investigated the early effects of a single dose of aflibercept, and of several other anti-VEGF/ VEGFR drugs, on MV generated by Ad-VEGF-A ${ }^{164}$. Acting within a few hours, all of these drugs induced extension of endothelial cell (EC) processes into and across MV lumens; these processes came to form transluminal bridges that led to MV collapse to form GMP, a progression that was accompanied by only modest EC death. MV collapse was quantified with a novel Evans blue dye (EBD) assay that provided a sensitive measure of declining intravascular volumes. We performed transcriptional profiling to identify genes whose expression was upregulated in the course of Ad-VEGF-A ${ }^{164}$-induced MV formation. One highly up-regulated gene, endothelial nitric oxide synthase (eNOS), was of particular interest. Inhibition of eNOS activity with $\mathrm{N}(\mathrm{G})$-nitro-L-arginine methyl ester (L-NAME) blocked VEGF-induced vascular permeability, prevented Ad-VEGF-A ${ }^{164}$-induced angiogenesis, inhibited B16F10 tumor growth, and, like anti-VEGF/VEGFR drugs, induced MV collapse to form GMP. Together these findings define the early effects of anti-VEGF/VEGFR drugs on newly formed angiogenic blood vessels, establish a role for eNOS as a potential therapeutic target, and have implications for understanding some of the mechanisms by which these drugs act in patients.

\section{Materials and Methods}

\section{Animals, Adenoviral Vectors, and Tumors}

Four- to 6-week-old female athymic nude mice were obtained from the National Cancer Institute (Bethesda, MD) and wild-type C57BL/6 and eNOS null mice from The Jackson Laboratory (Bar Harbor, ME). As previously described, ${ }^{6}$ an adenoviral vector engineered to express VEGF-A $^{164}$ (Ad-VEGF-A ${ }^{164}$ ) was injected into the ears of nude mice ( 1 to $5 \times 10^{7}$ plaque-forming units in $10 \mu \mathrm{L}$ of phosphate-buffered saline $3 \%$ glycerol) to induce angiogenesis. B16F10 melanoma cells $\left(1 \times 10^{6}\right.$ in Hanks' balanced salt solution) from ATCC (Manassas, VA) were injected subcutaneously in flanks or ears of nude mice or in flanks of eNOS knockout $\left(e \mathrm{NOS}^{-/-}\right)$mice and were harvested and weighed at indicated times. ${ }^{10}$ Miniosmotic pumps (model 1003D; delivering $1 \mu \mathrm{L} / \mathrm{h}$; Alzet Corp., Cupertino, CA) were filled with phosphate-buffered saline or L- or D-NAME and implanted subcutaneously in nude mice anesthetized with isoflurane. Mean arterial blood pressure was measured continuously in anesthetized nude mice before and after intraperitoneal injection of aflibercept with a carotid artery indwelling high-fidelity pressure catheter in the Beth Israel Deaconess Medical Center Cardiac Physiology Core as previously described. ${ }^{21}$ All animal studies were performed under protocols approved by the Beth Israel Deaconess Medical Center Institutional Animal Care and Use Committee.

\section{Drugs}

Aflibercept (VEGF Trap) and control peptide were gifts of Regeneron Pharmaceuticals, Tarrytown, NY, and aflibercept was available as Zaltrap (ziv-aflibercept). Combretastatin A4-phosphate was provided by Oxigene Inc. (Waltham, MA) or purchased from Sigma-Aldrich (St. Louis, MO). The receptor tyrosine kinase inhibitor sunitinib maleate (Pfizer, New York, NY) was from clinical supplies. G6 (an 
anti-VEGF antibody), ${ }^{22}$ DC101 (an antibody against VEGFR-2), ${ }^{23}$ and MF1 (an antibody against VEGFR-1) ${ }^{24}$ were provided by Eli Lilly and Company (Indianapolis, IN). L-NAME and its isomer D-NAME were obtained from Cayman Chemical Co. (Ann Arbor, MI). EBD and other chemicals were purchased from Sigma-Aldrich.

\section{Miles Assay}

Mice were anesthetized with isoflurane and injected i.v. with $0.1 \mathrm{~mL}$ of $0.5 \%$ EBD. Human recombinant VEGF-A (100 ng; National Cancer Institute) or Hanks' balanced salt solution (control) was then injected intradermally. Thirty minutes later, injection sites were photographed, collected in their entirety with 8-mm dermal punches, and weighed. EBD was extracted in formamide, and the amount of plasma albumin leakage was calculated as described for ear sites (see below).

\section{Quantification of Intravascular Plasma Volumes in Ad-VEGF-A ${ }^{164}$-Injected Ear Sites and Tumors}

The method used was modified from Nagy et al. ${ }^{7}$ Mice were anesthetized with isoflurane, and ears were photographed and then injected i.v. with EBD $(0.2 \mathrm{~mL} / 20$-g mouse of a $1.0 \%$ solution in saline). Eight minutes later, ears were photographed again, and $0.1 \mathrm{~mL}$ of blood was collected by retro-orbital puncture into $10 \mu \mathrm{L}$ of heparin. After an additional 2 minutes (ie, 10 minutes total EBD circulation time), mice were euthanized, and entire ear angiogenesis sites or tumors were collected with a 10-mm biopsy punch into $0.5 \mathrm{~mL}$ of formamide. Blood samples were centrifuged at $15,000 \times g$ for 10 minutes, and $10 \mu \mathrm{L}$ of recovered plasma was added to $0.5 \mathrm{~mL}$ of formamide. EBD was extracted at $56^{\circ} \mathrm{C}$ for 3 days for analysis by absorbance at $620 \mathrm{~nm}$. The concentration of EBD $(\mu \mathrm{g} / \mathrm{mL})$ in each tissue sample was determined with a standard curve, multiplied by $0.5 \mathrm{~mL}$ (the volume of formamide used for extraction) to obtain the micrograms of EBD in each tissue sample. The micrograms of EBD in each tissue sample was divided by the concentration of EBD in the plasma $(\mu \mathrm{g} / \mu \mathrm{L})$ of the respective mouse to calculate the volume of plasma $(\mu \mathrm{L})$ at the site.

\section{Isolation of RNA, Quantitative RT-PCR}

RNA was isolated with the Qiagen (Redwood City, CA) RNeasy Mini Kit and reverse transcribed using TaqMan Reverse Transcription Reagents (Applied Biosystems, Foster City, CA). Multigene transcriptional profiling was used to quantify mRNA as previously described. ${ }^{25}$

\section{Light and Electron Microscopy and Immunohistochemistry}

Ears were fixed in paraformaldehyde-glutaraldehyde and processed into Epon for 1- $\mu \mathrm{m}$ Giemsa-stained sections and for electron microscopy as previously described. ${ }^{3,6}$ For immunohistochemistry, tissues were fixed in $4 \%$ paraformaldehyde and processed for paraffin embedding and immunostaining as previously described. ${ }^{11,26}$ Primary rat anti-CD31 and rabbit anti-eNOS antibodies were from Novus Biologicals (Littleton, $\mathrm{CO}$ ) and biotinylated secondary anti-rat or anti-rabbit $\operatorname{IgG}$ antibodies from Vector Laboratories (Burlingame, CA). Reactions were amplified with vectastain $A B C$ reagent (Vector Laboratories) and visualized with $3,3^{\prime}$ diaminobenzidine peroxidase substrate solution. Sections were counterstained with methyl green.

\section{Statistical Analysis}

Data are presented as means \pm SEM. Statistical analysis was performed with Prism software version 5 (GraphPad Software, Inc., La Jolla, CA), using parametric tests where allowable. Two group comparisons used the parametric unpaired $t$-test with Welch correction, otherwise the nonparametric $U$-test was used. Multiple comparisons used analysis of variance with Dunnett's post test or nonparametric Kruskal-Wallis test.

\section{Results}

Single Injections of VEGF/VEGFR Antagonists Induce Rapid MV Collapse to Form GMP

Five days after ear injection with Ad-VEGF-A ${ }^{164}$, at the height of the MV response, ${ }^{1,6}$ mice were injected with a single $25-\mathrm{mg} / \mathrm{kg}$ dose of aflibercept or control peptide. ${ }^{27}$ Two, 6, and 24 hours later ears were photographed (Figure 1, A-C) and harvested for light and electron microscopy (Figure 1). Similar results were obtained whether aflibercept was administered by s.c., i.p., or i.v. routes. Within hours, the EC lining MV extended cytoplasmic processes into and across MV lumens (Figure 1, E-H, N and O); subsequently, these processes became bridges, dividing MV lumens into multiple, small channels, many barely sufficient to admit single red blood cells (Figure $1, \mathrm{I}-\mathrm{L}$ and $\mathrm{P}-\mathrm{R}$ ). As MV collapsed, their thin, flattened lining ECs became rounded and developed large nuclei with prominent nucleoli, features characteristic of activated cells. Only a minority of ECs exhibited signs of injury (Figure 1, K and $\mathrm{P}-\mathrm{R}$ ). MV in control peptide-injected mice persisted unchanged, just as in untreated mice (Figure 1, D and M).

In contrast to $\mathrm{MV}$, the other vessel types present (capillaries, developing FAs, draining veins) were unaffected by aflibercept (not shown). Treatment with aflibercept at later intervals (30 to 71 days) after Ad-VEGF$\mathrm{A}^{164}$ injection caused no discernable effect on any of the blood vessels present (not shown). These findings are consistent with our earlier results that had demonstrated that a multiday course of aflibercept treatment had little or no effect on blood vessels other than MV. ${ }^{19}$ 

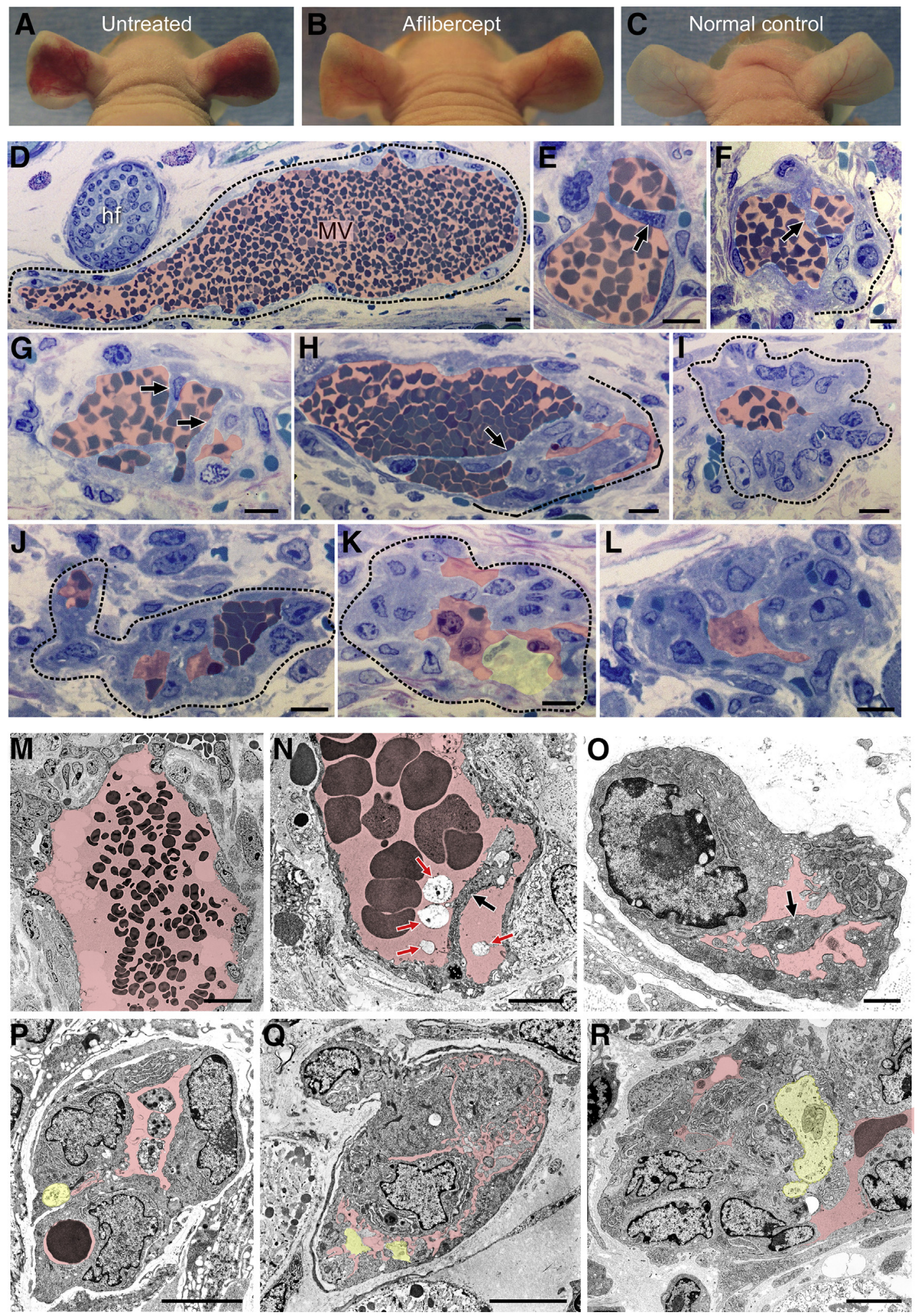

Figure 1 Aflibercept induces rapid collapse of mother vessels (MV) to glomeruloid microvascular proliferations (GMP). A-C: Nude mouse ears 6 days after injection of $10^{7}$ plaque-forming units of adenovirus expressing vascular endothelial growth factor (VEGF)-A (Ad-VEGF-A ${ }^{164}$ ); mice were left untreated or were treated s.c. with a single dose of aflibercept $(25 \mathrm{mg} / \mathrm{kg}$ ) or control peptide 24 hours earlier on day 5. D-R: Light (D-L) and electron (M-R) microscopic images of ears injected 5 days earlier with Ad-VEGF- $\mathrm{A}^{164}$ and harvested 6 hours after treatment with control peptide (D and M; typical MV) or aflibercept (all others). Transluminal endothelial cell (EC) bridging (E-H, $\mathbf{N}$, and $\mathbf{0}$; black arrows), proceeding to MV collapse and formation of typical GMP (I-L and $\mathbf{P}-\mathbf{R})$. Most ECs are healthy with prominent activated nuclei; occasional dead ECs are indicated with yellow shading ( $\mathbf{K}$ and $\mathbf{P}-\mathbf{R})$. Pink shading indicates vascular lumens; red arrows $(\mathbf{N})$, cellular debris within vascular lumens; dashed lines, an $M V$ (D) and GMP formation (F and $\mathbf{H}-\mathbf{K}) . n>50$ mice. Scale bars: $10 \mu \mathrm{m}(\mathbf{D}-\mathbf{L}) ; 20 \mu \mathrm{m}(\mathbf{M}) ; 5 \mu \mathrm{m}(\mathbf{N}$ and $\mathbf{P}-\mathbf{R}) ; 1 \mu \mathrm{m}(\mathbf{0})$. hf, hair follicle. 

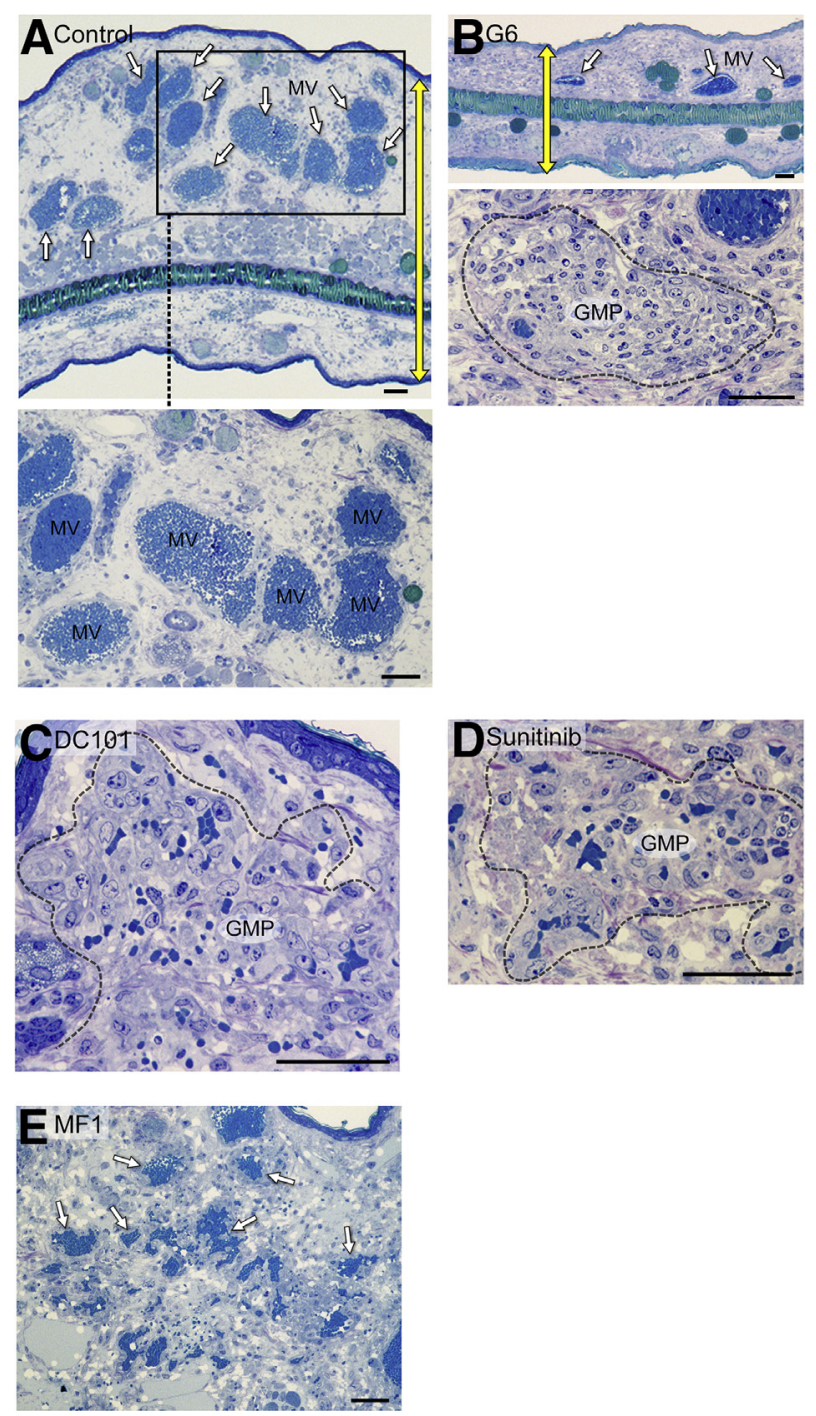

Figure 2 Effects of several vascular endothelial growth factor (VEGF)/ VEGF receptor (VEGFR) antagonists on adenovirus expressing VEGF-A (AdVEGF- ${ }^{164}$ )-induced mother vessels (MV). Five days after Ad-VEGF-A ${ }^{164}$ ear injection, nude mice were treated with single treatments of the following: phosphate-buffered saline (PBS) (A); G6 (20 mg/kg), an antibody against VEGF-A (B); DC101 $(40 \mathrm{mg} / \mathrm{kg})$, an antibody against VEGFR-2 (C); sunitinib $(50 \mathrm{mg} / \mathrm{kg})$, a tyrosine kinase inhibitor with activity against VEGFR-2 (D); MF1 $(40 \mathrm{mg} / \mathrm{kg})$, an antibody against VEGFR-1 (E). The boxed area in A indicates MV field and is shown at higher magnification in the bottom panel. Sunitinib was given by gavage, the other drugs were given i.p. Ears were taken for histologic examination 24 hours later. G6, DC101, and sunitinib all induced MV collapse to glomeruloid microvascular proliferations (GMP), similar to that induced by aflibercept, whereas MV persisted in mice treated with PBS and MF1. Note reduction in edema indicated by yellow doubleheaded arrows, indicating ear thickness in untreated control $(A)$ versus G6-treated (B) mice. White arrows indicate MV in A, the top panel in $\mathbf{B}$, and E. Dashed lines indicate well developed GMP (B, C, and D). Scale bars: 100 $\mu \mathrm{m}$ (A and $\mathbf{B}$, top panel); $50 \mu \mathrm{m}$ (B, bottom panel and $\mathbf{C}-\mathbf{E})$.

We also treated mice 5 days after receiving Ad-VEGF$\mathrm{A}^{164}$ ear injections with single treatments of the receptor tyrosine kinase inhibitor sunitinib or with antibodies against VEGF-A (G6) or against VEGF receptor 2 (DC101). Like aflibercept, these drugs caused MV
(Figure 2A) to collapse to form GMP, accompanied by only modest EC injury (Figure 2, B-D). In contrast, MV persisted in mice treated with MF1, an antibody against VEGF receptor 1 (Figure 2E).

\section{Single Injections of Combretastatin CA4 Selectively Kill MV ECs}

Combretastatins are tubulin-binding phenols that disrupt tumor blood vessels. ${ }^{28}$ To determine their effects on MV, mice were treated with a single i.p. injection of combretastatin CA4 5 days after ear injection with Ad-VEGF-A ${ }^{164}$. Unlike aflibercept and the other VEGF/VEGFR-targeting drugs tested, combretastatin CA4 caused dramatic killing of MV ECs within 2 hours, and by 6 hours MV were largely obliterated (Figure 3). The vascular damage induced by combretastatin CA4 was selective for MV, as other vessel types were spared (not shown). When administered at late intervals (30 to 71 days) after Ad-VEGF-A ${ }^{164}$ injection, combretastatin CA4 caused no discernible blood vessel damage.

\section{EBD Assay to Quantify the Effects of Treatment on Vascular Volumes at Angiogenic Sites}

The collapse of MV induced by aflibercept, sunitinib, and antibodies against VEGF-A and VEGFR-2 resulted in striking reductions in local vascular volumes as judged by light and electron microscopy (Figures 1 and 2). To substantiate these changes, we devised a quantitative assay with $\mathrm{EBD}$, a low molecular weight dye that binds to plasma albumin and so provides a faithful tracer of intravascular plasma volumes. The antiangiogenic effects of a single injection of aflibercept were visible macroscopically both before and after EBD injection (Figure 4A).

To quantify these effects, we performed punch biopsies that encompassed entire angiogenic sites 10 minutes after EBD injection, that is, at a time that allowed filling of the angiogenic vasculature with minimal dye leakage from hyperpermeable MV. ${ }^{1,7}$ A single injection of aflibercept profoundly reduced EBD accumulation at 5-day Ad-VEGF-A ${ }^{164}$ injection sites (Figure 4B); within 2 hours, plasma volumes were reduced by nearly half, and at 24 hours by $>75 \%$. Antibodies against VEGF and VEGFR-2, as well as sunitinib, also reduced plasma volumes at 24 hours by $64 \% \pm 22 \%, 29 \% \pm 19 \%$, and $22 \% \pm 10 \%$, respectively. MF1 did not reduce plasma volumes significantly. Combretastatin CA4 also reduced plasma volumes significantly (Figure $4 \mathrm{C}$ ) but did so by a very different mechanism (Figures 1, 2, 3, and 4). Neither aflibercept nor combretastatin CA4 significantly affected vascular plasma volumes when administered 71 days after Ad-VEGF-A ${ }^{164}$ injection (Figure 4, B and C).

\section{Role of eNOS in Ad-VEGF-A ${ }^{164}$-Induced Angiogenesis}

eNOS is induced by $\mathrm{VEGF}^{29-31}$ and is highly expressed in many tumors. ${ }^{23,31-33}$ The product of eNOS, nitric oxide 

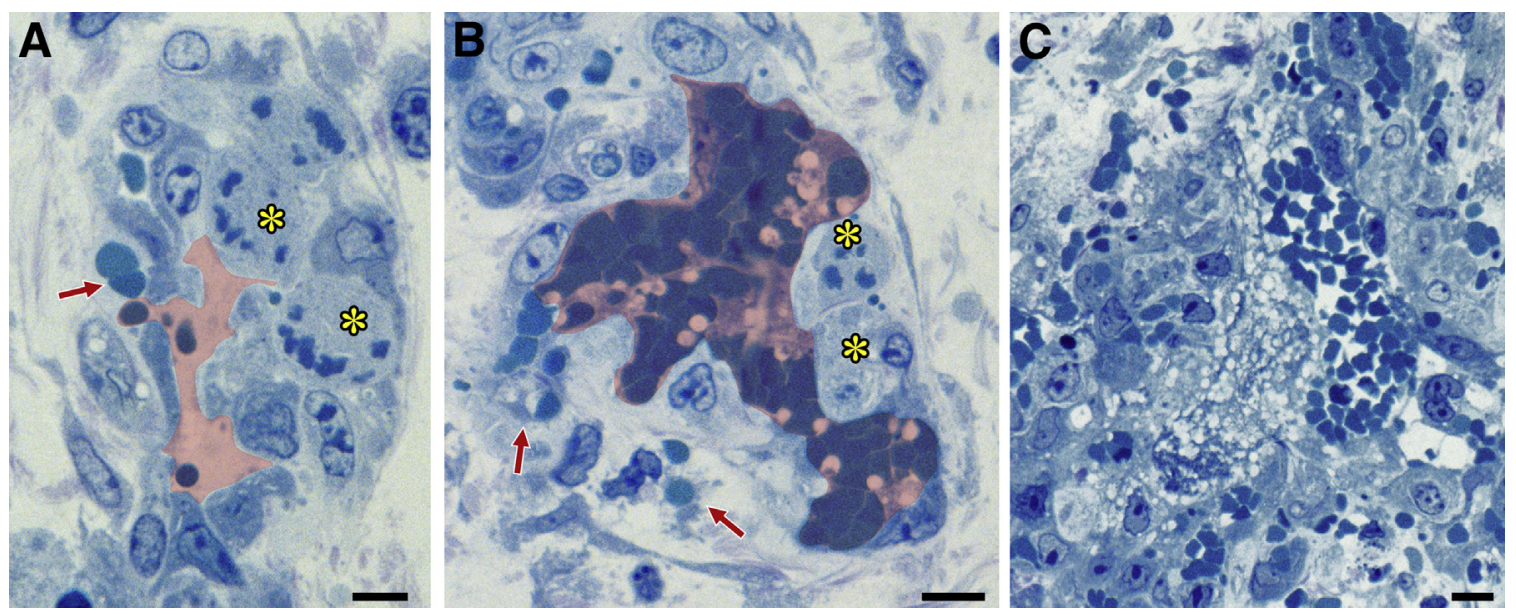

Figure 3 Effects of combretastatin CA4 on adenovirus expressing vascular endothelial growth factor (VEGF)-A (Ad-VEGF-A $\mathrm{A}^{164}$ )-induced mother vessels (MV). Five days after injection of $10^{7}$ plaque-forming units of Ad-VEGF-A $A^{164}$ nude mice were treated with combretastatin CA4. A and B: MV exhibit endothelial cell (EC) necrosis (asterisks) and red blood cell extravasation (red arrows) 2 hours after i.p. injection of $6.25 \mathrm{mg}$ of combretastatin CA4. Pink shading demarcates vascular lumens. C: At 6 hours, MV are obliterated and replaced by focal hemorrhage, fibrin, and inflammatory cells. Scale bars: $10 \mu \mathrm{m}(\mathbf{A}-\mathbf{C})$.

(NO), has been implicated in VEGF-induced permeability and angiogenesis. ${ }^{29-31,34}$ With the use of the Miles assay, we found that L-NAME, a potent eNOS inhibitor, strongly inhibited VEGF-induced vascular permeability in mouse flank skin (Supplemental Figure S1).

To determine whether eNOS might have a role in AdVEGF- $\mathrm{A}^{164}$-induced MV formation and devolution, we performed RT-PCR on RNA isolated from ear angiogenic sites taken at early intervals after Ad-VEGF-A ${ }^{164}$ injection, that is, at times paralleling MV development. eNOS mRNA expression increased progressively from day 1 , reaching a maximum on day 5 at $>14$ times control levels (Figure 5A). By contrast, inducible NOS and neuronal NOS were expressed at insignificant levels at all time points $(<2$

A
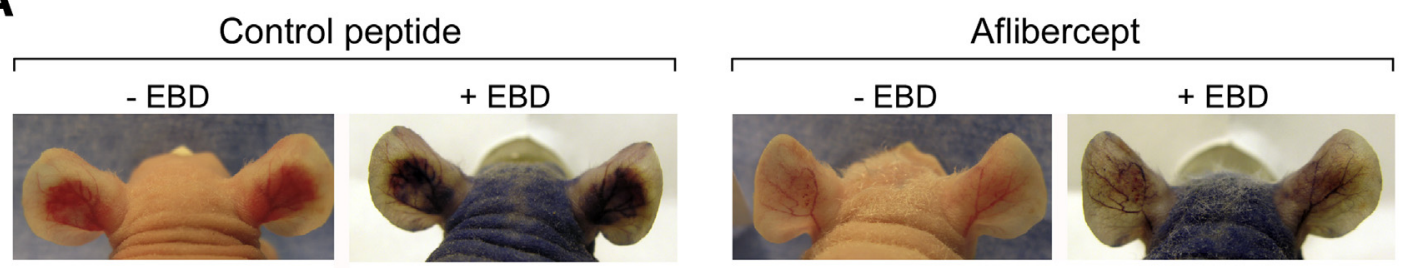

B

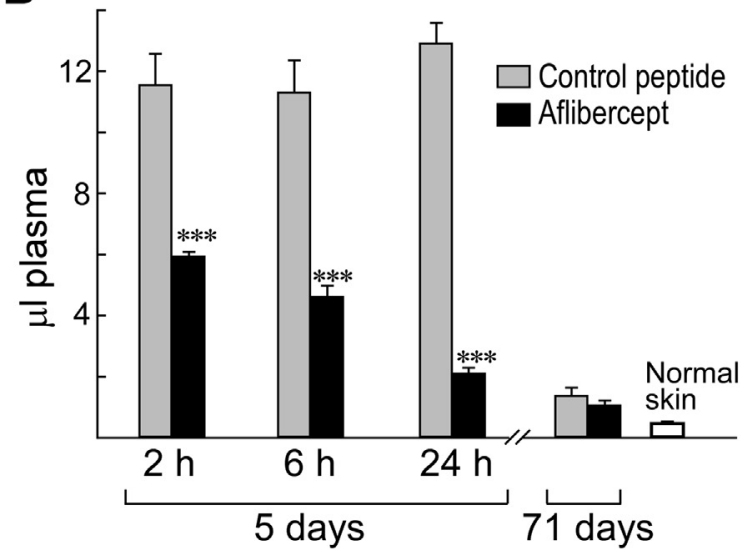

C

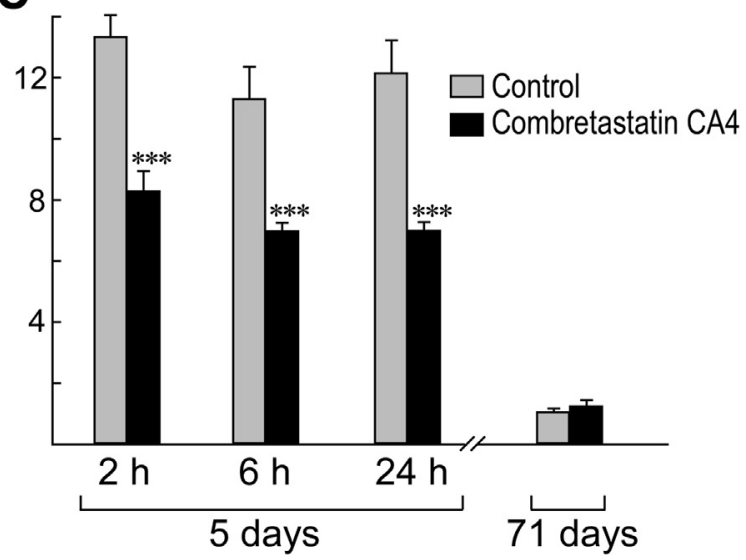

Figure 4 Evans blue dye (EBD) assay for quantifying effects of aflibercept and combretastatin CA4 on vascular plasma volumes at angiogenic sites induced by adenovirus expressing vascular endothelial growth factor (VEGF)-A (Ad-VEGF-A ${ }^{164}$ ). A: Nude mouse ears 6 days after ear injection with Ad-VEGF-A $\mathrm{A}^{164} \pm 30$ minutes after injection of EBD. Mice had been treated 1 day earlier (day 5) with control peptide (left panels) or with aflibercept (right panels). Note striking reduction in angiogenesis and EBD accumulation induced by aflibercept versus control peptide. B and C: Local plasma volumes ( $\mu \mathrm{L})$ at indicated intervals after single injections of aflibercept (25 mg/kg s.c.) or combretastatin CA4 (6.25 mg i.p.) at 5 or 71 days after Ad-VEGF-A ${ }^{164}$ ear injection. Vascular volume of normal ear skin is shown for comparison. $n=8$ to 16 mice per group. ${ }^{* * *} P<0.001$ versus control (U-test). 
and $<0.6$ mRNA copies $/ 10^{6}$ copies 18S RNA, respectively). ${ }^{25}$ Immunohistochemistry demonstrated that MV ECs expressed eNOS protein strongly compared with normal microvascular ECs (Figure 5B). Further, inhibition of eNOS activity by L-NAME $(40 \mathrm{mg} / \mathrm{kg}$ in drinking water begun 2 days before Ad-VEGF-A ${ }^{164}$ injection) prevented angiogenesis and associated increased MV permeability as observed macroscopically (Figure 5C) and as confirmed by EBD measures of vascular volumes (Figure 5D). L-NAME also inhibited the growth of B16F10 melanomas in nude mice (Figure 5E), and B16F10 tumor cells grew more slowly in $e \mathrm{NOS}^{-/-}$mice compared with wild-type C57B1/6 mice (Figure 5F).

\section{Effect of L-NAME on Ad-VEGF-A ${ }^{164}$-Induced MV}

Because L-NAME inhibited MV formation, we considered the possibility that, like aflibercept and the other drugs targeting the VEGF/VEGFR axis, it might also induce MV collapse to GMP. To test this possibility, we treated mice with L-NAME (or with its inactive isomer D-NAME) 5 days after their ears had been injected with Ad-VEGF-A ${ }^{164}$, harvesting them 1 day later. Because oral administration could not be counted on to deliver a consistent amount of drug reliably over a short (1 day) period of time, L- and D-NAME were administered by way of s.c. implanted minipumps. L-NAME, but not D-NAME, caused MV bridging and collapse to form GMP (Figure 6, A-C), accompanied by significant reductions in local intravascular volumes (Figure 6D). L-NAME had less or no effect on vascular volumes at later times (12 to 60 days) after Ad-VEGF-A ${ }^{164}$ injection. L-NAME also rapidly reduced vascular volumes in B16F10 melanomas growing in nude mouse ears (Figure 6E).

\section{Aflibercept Induces Hypertension}

Anti-VEGF/VEGFR drugs commonly induce hypertension in cancer patients. ${ }^{35-40}$ Because hypertension results from systemic arterial contraction, the hypertension induced by these drugs might result from inhibiting eNOS and so reducing expression of its product, the vasodilator NO.
A

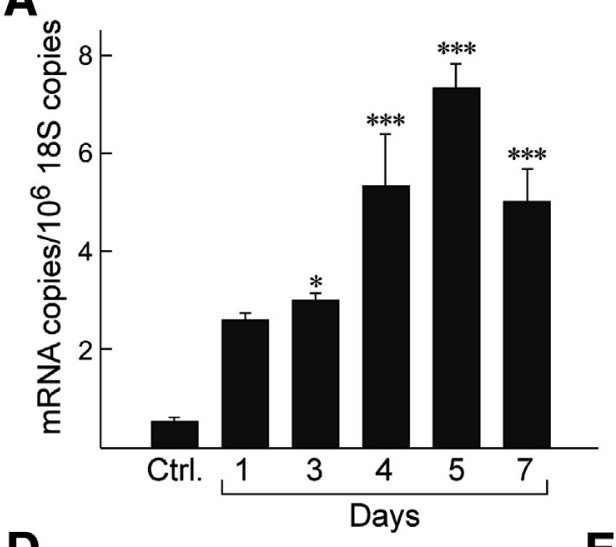

D

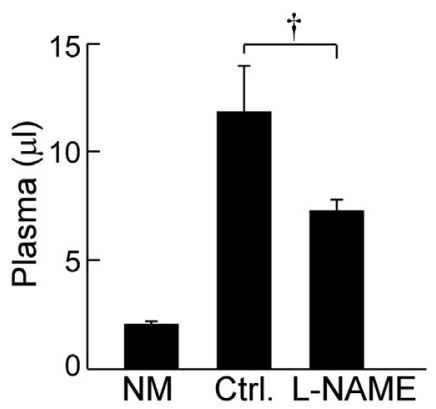

B

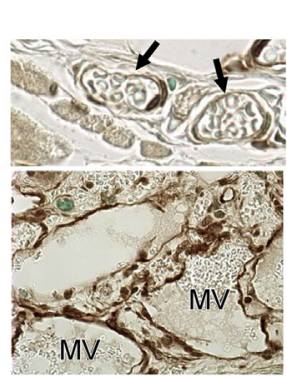

E

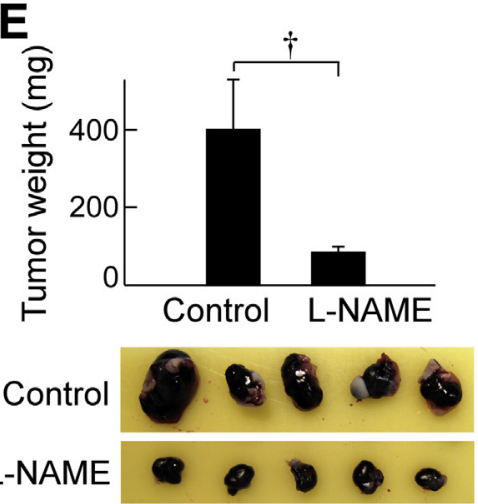

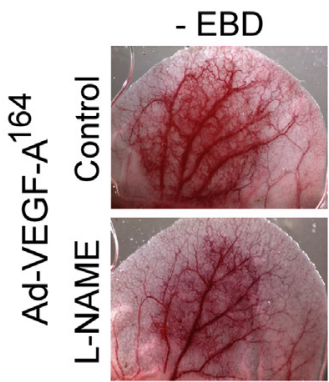

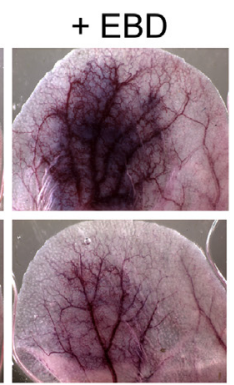

$\mathbf{F}$

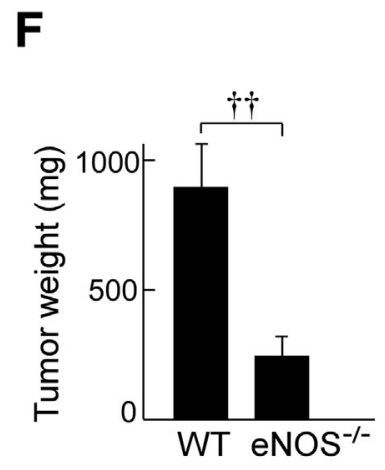

Figure 5 eNOS induction and endothelial nitric oxide synthase (eNOS) inhibition by N(G)-nitro-L-arginine methyl ester (L-NAME) in adenovirus expressing vascular endothelial growth factor (VEGF)-A (Ad-VEGF-A ${ }^{164}$ )-induced angiogenesis and in B16F10 tumor growth. A: Induction of eN0S mRNA in ear skin after injection of Ad-VEGF-A ${ }^{164}$. B: Immunohistochemistry demonstrates strong eNOS staining of ear mother vessels (MV) 5 days after injection of Ad-VEGF-A ${ }^{164}$ (bottom panel) compared with minimal staining of microvessels in normal ears (arrows; top panel). C: Macroscopic view of nude mouse ears 5 days after injection of Ad-VEGF-A ${ }^{164}$ in mice receiving L-NAME or control in their drinking water \pm i.v. Evans blue dye (EBD) injection. D: Quantification of the effects of L-NAME $\left(40 \mathrm{mg} / \mathrm{kg}\right.$ in drinking water) on vascular volumes ( $\mu \mathrm{L}$ plasma) at angiogenic sites 5 days after injection of Ad-VEGF-A ${ }^{164}$. E: Growth of B16F10 melanoma cells s.c. in flank skin of nude mice receiving L-NAME in their drinking water. F: B16F10 melanoma cells grow more slowly in eNOS ${ }^{-/-}$mice than in wild-type (WT) C57Bl/ 6 eNOS ${ }^{+/+}$control mice. $n=8$ mice per group (D-F). ${ }^{\star} P<0.05, * * * P<0.001$ versus Ctrl (analysis of variance with Dunnett's multiple comparison post test; $\mathbf{A}) ;{ }^{\dagger} P<0.05 ;{ }^{\dagger \dagger} P<0.01$ (U-test; D-F). Original magnification: $\times 40$ (B). Ctrl, untreated; NM, normal skin. 
A

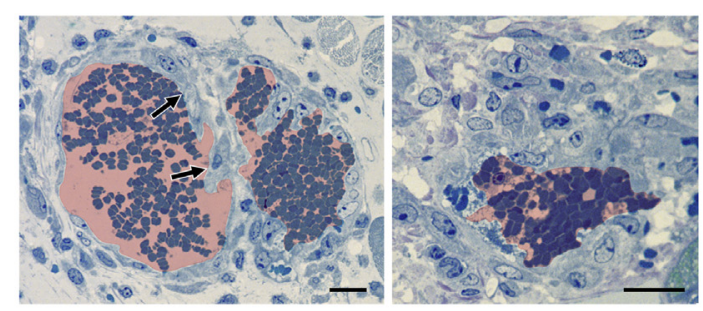

D

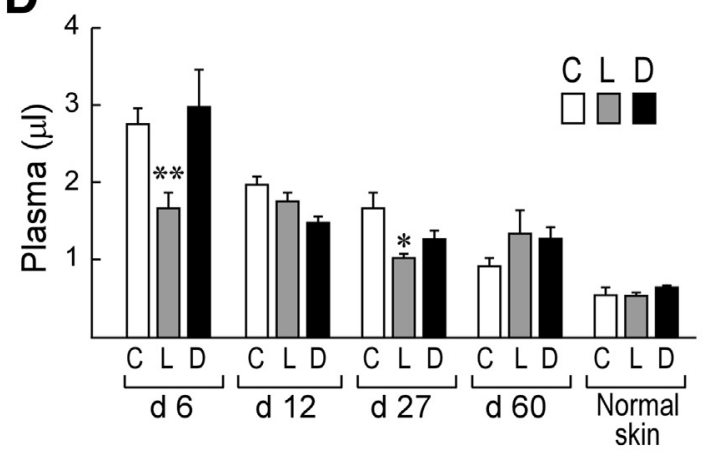

C

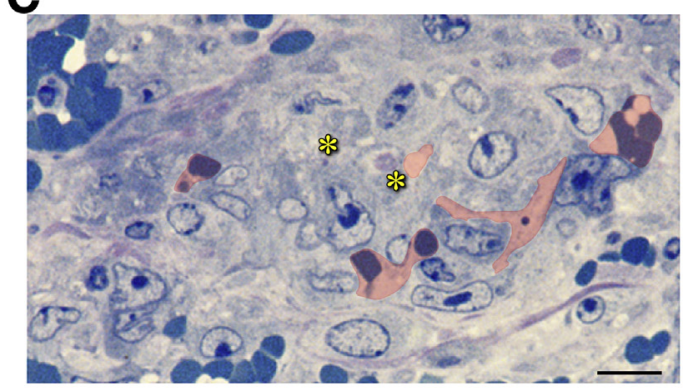

$\mathbf{E}$

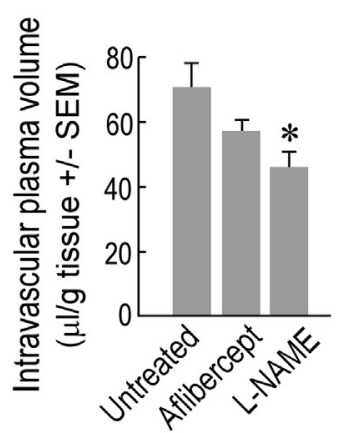

Figure 6 Effects of N(G)-nitro-L-arginine methyl ester (L-NAME) on mother vessels (MV). A-C: Minipumps delivering L-NAME (134 mg/kg per day) were implanted in nude mice 5 days after ear injection with adenovirus expressing vascular endothelial growth factor (VEGF)-A (Ad-VEGF-A ${ }^{164}$ ). Twenty-four hours later ears were harvested for histologic examination. Note MV bridging (arrows) and collapse to form glomeruloid microvascular proliferations (GMP), accompanied by occasional cell death (asterisks). Pink shading demarcates vascular lumens. D: Minipumps delivering control phosphate-buffered saline (PBS) (C) or $134 \mathrm{mg} / \mathrm{kg}$ per day of L-NAME (L) or D-NAME (D) were implanted s.c. in nude mice whose ears had been injected with Ad-VEGF-A ${ }^{164} 5,11,26$, or 59 days earlier. Twenty-four hours after minipump implant mice were injected i.v. with Evans blue dye (EBD) and were harvested 10 minutes later to measure vascular volumes. E: Tumors in nude mouse ears 13 days after injection of $10^{6}$ B16F10 melanoma cells. Mice were then left untreated, injected with aflibercept i.p., or received s.c. minipumps delivering $134 \mathrm{mg} / \mathrm{kg}$ per day L-NAME. Twenty-four hours later, mice were injected i.v. with EBD, and entire tumors were harvested with punch biopsies to measure plasma volumes. $n=10$ to 39 mice per group (D); $n=8$ mice per group (E). ${ }^{*} P<0.05,{ }^{* *} P<0.01$ (Kruskal-Wallis multiple comparisons test) versus control (D) or versus untreated (E). Scale bars: $20 \mu \mathrm{m}$ (A and B); $10 \mu \mathrm{m}$ (C).

Consistent with this possibility, aflibercept, at the doses used to induce MV collapse, induced a significant increase in mean arterial blood pressure within minutes that could be prevented with the NO donor nitroglycerin, as well as by a calcium channel blocker, diltiazem (Figure 7).

\section{Discussion}

Although widely used in treating cancer, the actions of antiangiogenesis drugs are not well understood. ${ }^{14}$ The general assumption has been that these drugs kill tumor blood vessel ECs and so compromise tumor blood vessel function. ${ }^{41}$ With the use of the Ad-VEGF-A ${ }^{164}$ model, we now demonstrate that these drugs can also exert important effects on angiogenic blood vessels that are largely independent of direct EC killing. Within hours a single dose of aflibercept, and of several other anti-VEGF/VEGFR drugs, transformed MV into GMP by a process that began with transluminal EC bridging and that was followed by MV collapse (Figures 1 and 2). Thus, by acutely inhibiting VEGF-A signaling, these drugs rapidly reversed the earliest step in VEGF-induced angiogenesis, MV formation, and returned MV to the normal capillaries and venules from which they arose with GMP as an intermediate step (Figure 8). They thus greatly accelerated a process that required weeks in the absence of treatment as VEGF-A levels declined gradually in parallel with the stochastic discharge of VEGF-A ${ }^{164}$-expressing adenovirus from infected cells. ${ }^{1,3,6,11} \mathrm{MV}$ are thus seen to be unstable blood vessels that require high levels of VEGF-A for both

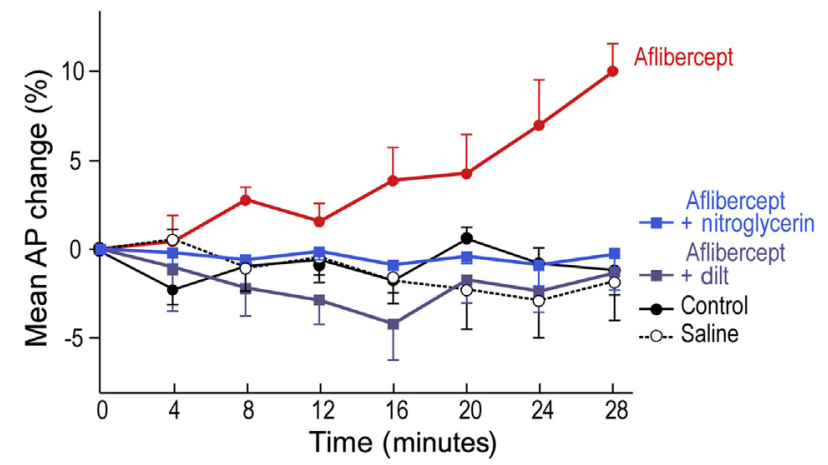

Figure 7 Aflibercept (25 mg/kg i.p.) induces significant hypertension by 16 minutes in nude mice that is prevented by i.v. nitroglycerin $(20 \mu \mathrm{g} /$ $\mathrm{kg}$ per minute) and by diltiazem (dilt; $10 \mu \mathrm{g} / \mathrm{kg}$ per minute). $n=5$ to 7 mice per group. Analysis of variance with Dunnett's multiple comparison test. AP, arterial pressure. 
generation and maintenance; not surprisingly, therefore, they are the selective targets of anti-VEGF/VEGFR drugs. Another drug in clinical trials, the vasodisruptive drug combretastatin CA4, ${ }^{28,42}$ also targeted MV selectively, but it acted in a very different manner from anti-VEGF/VEGFR drugs, causing rapid and extensive MV EC necrosis and vessel obliteration (Figure 3).

GMP are prominent in glioblastoma multiforme and are a minority component in many other human cancers, ${ }^{43-45}$ but the mechanisms by which they form have been obscure. ${ }^{11}$ Our findings suggest that GMP may form in tumors by a mechanism similar to that in the Ad-VEGF-A ${ }^{164}$ setting, that is, from MV in zones of locally diminished VEGF-A signaling. Our findings also demonstrate that GMP can develop in the absence of tumor cells and so exclude the necessity of an alternatively proposed mechanism in which GMP form from the pulling together of capillary ECs by tumor cells. ${ }^{46}$

Histologic examination and electron microscopy predicted that aflibercept and the other anti-VEGF/VEGFR drugs tested would substantially reduce vascular volumes as MV collapse to form GMP (Figures 1 and 2). To test this prediction we developed a novel quantitative assay using EBD. Aflibercept (Figure 4), G6 (an anti-VEGF antibody), sunitinib (a tyrosine kinase inhibitor), and DC101 (an antibody against VEGF receptor 2), all induced striking reductions in local vascular volumes within 24 hours, whereas MF1 (an antibody targeting VEGF receptor 1) did not. These data are in accord with our microscopic results (Figures 1 and 2) and with literature findings that VEGF-A induces angiogenesis primarily through VEGF receptor 2, not VEGF receptor $1 .^{5}$ The EBD assay can have use in a wider context for quantifying the effects of antiangiogenesis drugs on tumor vasculature. Current methods for doing so generally make use of immunohistochemistry to count the numbers, shapes, or sizes of CD31-stained blood vessels in

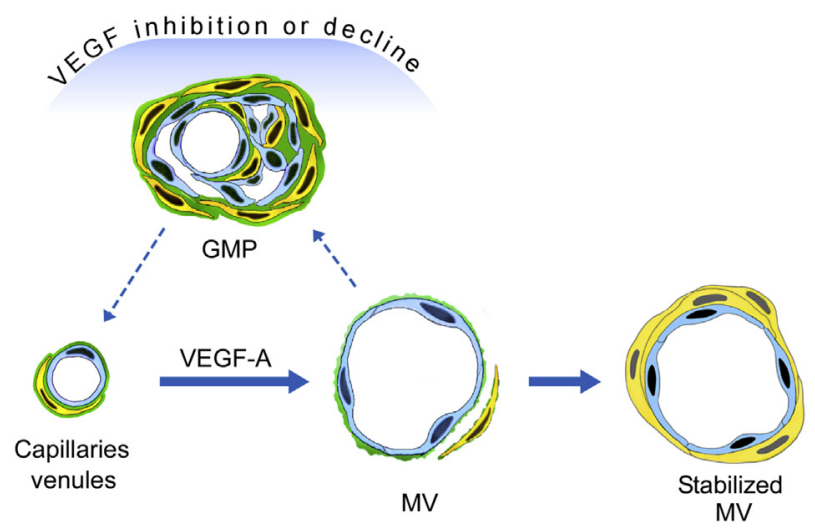

Figure 8 Schematic diagram depicting the induction of mother vessels (MV) and stabilized MV from normal capillaries and venules by adenovirus expressing vascular endothelial growth factor (VEGF)-A (Ad-VEGF-A ${ }^{164}$ ). MV, but not stabilized MV, revert within hours to normal microvessels by way of glomeruloid microvascular proliferations (GMP) after VEGF inhibition by anti-VEGF/VEGF receptor (VEGFR) drugs or as VEGF levels decline over days to weeks in the absence of treatment as adenovirus is discharged stochastically from infected cells. paraffin-embedded tissue sections. ${ }^{40,47}$ This method has important limitations. It is at best semiquantitative and is subject to sampling error because of the extensive variation in vascular density in different portions of a single tumor. In contrast, the EBD assay can quantify the vascular volume of an entire tumor (Figure 6E). Combretastatin CA4 also reduced local vascular volumes strikingly, but it did so by obliterating MV rather than by causing MV collapse to GMP (Figure 3). Therefore, it remains important to correlate EBD measurements with histologic examination for understanding the mechanisms of antivascular drug action (Figures 1, 2, 3, and 4).

An extensive literature has implicated eNOS and its product NO in tumor blood vessel formation and function. . $^{1,23,31-33}$ We have shown here that $e N O S$ expression is increased at both the RNA and protein levels in parallel with Ad-VEGF$\mathrm{A}^{164}$-induced MV formation (Figure 5, A and B) and have confirmed earlier findings $^{34}$ that L-NAME, a selective eNOS inhibitor, blocked VEGF-A-induced vascular permeability in the Miles assay (Supplemental Figure S1). We have now also shown that L-NAME largely prevents MV formation when treatment is initiated before and continued daily after Ad-VEGF-A ${ }^{164}$ injection (Figure 5, C and D). Consistent with our hypothesis, L-NAME also inhibited the growth of B16F10 tumors (Figure 5E), and these tumors grew less well in $e \mathrm{NOS}^{-/-}$mice than in wild-type mice (Figure 5F). Finally, like aflibercept and the other anti-VEGF/VEGFR drugs tested here, L-NAME, but not D-NAME, induced MV involution to GMP with an accompanying reduction in vascular volumes within 24 hours (Figure 6, A-D).

The finding that L-NAME mimics the action of antiVEGF/VEGFR drugs suggests that these drugs might induce MV collapse by inhibiting eNOS. ${ }^{23}$ Inhibition of eNOS activity by anti-VEGF/VEGFR drugs or by L-NAME is expected to diminish generation of its product, the potent vasodilator NO, leading to contraction of smooth muscle-lined blood vessels such as FAs. FA contraction would reduce blood flow to downstream MV. MV, lined only by ECs and lacking smooth muscle cell support, are expected to depend on blood flow for maintaining patency; in other settings blood flow is necessary for maintaining vascular channels that are lined only by ECs. ${ }^{48}$ Consistent with this possibility, aflibercept at the doses used here to induce MV collapse induced a rapid (within 16 minutes) increase in mean arterial blood pressure (Figure 7), indicative of systemic arterial contraction; aflibercept-induced hypertension was prevented by the NO donor nitroglycerin. Similarly, cancer patients treated with anti-VEGF/ VEGFR drugs commonly develop hypertension, a result that has been correlated with therapeutic response. ${ }^{35-40}$ Thus, our data provide evidence for a hypothesis suggested by Ellis and Hicklin, ${ }^{14}$ namely, that anti-VEGF/ VEGFR drugs can act, at least in part, by causing arterial constriction that compromises downstream blood flow.

Our findings also offer an explanation for the state of vascular normalization that follows antiangiogenic therapy 
in both cancer patients and tumor-bearing mice. ${ }^{49,50}$ It has long been known that the tumor vasculature is hyperpermeable to plasma proteins (reviewed in Dvorak ${ }^{4}$ ) and that the resulting vascular leakage leads to the edema and increased interstitial pressure characteristic of tumor stroma. ${ }^{49}$ However, for a time after receiving anti-VEGF/ VEGFR therapy, all of these abnormalities are reversed, and blood vessels appear more normal by histologic examination and radiologic imaging. ${ }^{49,50}$ Because MV, and to a much lesser extent GMP, are the only hyperpermeable tumor blood vessel subsets, ${ }^{1,11}$ a return to vascular normality is what would be expected from treatments that cause abnormal, hyperpermeable MV to revert to GMP and subsequently to normal capillaries and venules (Figure 8).

Finally, because anti-VEGF/VEGFR drugs act selectively on $\mathrm{MV}$, our findings predict that their efficacy in treating cancer will depend on the numbers of MV present. Consistent with this prediction, rapidly growing mouse tumors with large numbers of MV are commonly susceptible to these drugs. The situation is different for more slowly growing mouse tumors that have had time to develop an established vasculature, ${ }^{16}$ and even more so for human cancers that typically develop over months or years before discovery. In such tumors, MV typically account for a smaller fraction of the tumor vasculature, because all but the most recently formed blood vessels have had time to acquire a smooth muscle coat that makes them resistant to anti-VEGF/VEGFR therapy. Developing methods to quantify MV in tumors could be useful in predicting the likelihood of success of anti-VEGF/VEGFR therapy in individual cancer patients.

\section{Acknowledgments}

B.S., L.E.B., R.S.B., A.M.D., J.A.N., and H.F.D. conceived and designed experiments; S.C., E.M., M.S., and J.A.N., performed experiments involving endothelial nitric oxide synthase; B.S., T.E.S., and A.M.D. performed light and electron microscopy; P.M.K. performed hypertension experiments; S.-C.J. performed multigene transcriptional profiling; B.S. performed all other experiments; H.F.D. wrote the manuscript with input from all of the other authors.

\section{Supplemental Data}

Supplemental material for this article can be found at http://dx.doi.org/10.1016/j.ajpath.2017.06.010.

\section{References}

1. Nagy JA, Feng D, Vasile E, Wong WH, Shih SC, Dvorak AM, Dvorak HF: Permeability properties of tumor surrogate blood vessels induced by VEGF-A. Lab Invest 2006, 86:767-780

2. Fu Y, Nagy JA, Dvorak AM, Dvorak HF: Tumor blood vessels: structure, function, and classification. Edited by Teicher B, Ellis L. In
Cancer Drug Discovery and Function Antiangiogenic Agents in Cancer Therapy. Totowa, NJ: Humana Press, 2007. pp. 205-224

3. Nagy JA, Dvorak HF, Dvorak AM: VEGF-A and the induction of pathological angiogenesis. Annu Rev Pathol 2007, 2:251-275

4. Dvorak HF: Rous-Whipple Award Lecture. How tumors make bad blood vessels and stroma. Am J Pathol 2003, 162:1747-1757

5. Shibuya M, Claesson-Welsh L: Signal transduction by VEGF receptors in regulation of angiogenesis and lymphangiogenesis. Exp Cell Res 2006, 312:549-560

6. Pettersson A, Nagy JA, Brown LF, Sundberg C, Morgan E, Jungles S, Carter R, Krieger JE, Manseau EJ, Harvey VS, Eckelhoefer IA, Feng D, Dvorak AM, Mulligan RC, Dvorak HF: Heterogeneity of the angiogenic response induced in different normal adult tissues by vascular permeability factor/vascular endothelial growth factor. Lab Invest 2000, 80:99-115

7. Nagy JA, Shih SC, Wong WH, Dvorak AM, Dvorak HF: Chapter 3. The adenoviral vector angiogenesis/lymphangiogenesis assay. Methods Enzymol 2008, 444:43-64

8. Paku S, Paweletz N: First steps of tumor-related angiogenesis. Lab Invest 1991, 65:334-346

9. Warren B: The vascular morphology of tumors. Edited by Peterson H-I. In Tumor Blood Circulation: Angiogenesis, Vascular Morphology and Blood Flow of Experimental and Human Tumors. Boca Raton, FL: CRC Press, 1979. pp. 1-47

10. Zeng H, Qin L, Zhao D, Tan X, Manseau EJ, Van Hoang M, Senger DR, Brown LF, Nagy JA, Dvorak HF: Orphan nuclear receptor TR3/Nur77 regulates VEGF-A-induced angiogenesis through its transcriptional activity. J Exp Med 2006, 203:719-729

11. Sundberg C, Nagy JA, Brown LF, Feng D, Eckelhoefer IA, Manseau EJ, Dvorak AM, Dvorak HF: Glomeruloid microvascular proliferation follows adenoviral vascular permeability factor/vascular endothelial growth factor-164 gene delivery. Am J Pathol 2001, 158:1145-1160

12. Jain RK: Lessons from multidisciplinary translational trials on antiangiogenic therapy of cancer. Nat Rev Cancer 2008, 8:309-316

13. Wang X, Solban N, Khanna P, Callea M, Song J, Alsop DC, Pearsall RS, Atkins MB, Mier JW, Signoretti S, Alimzhanov M, Kumar R, Bhasin MK, Bhatt RS: Inhibition of ALK1 signaling with dalantercept combined with VEGFR TKI leads to tumor stasis in renal cell carcinoma. Oncotarget 2016, 7:41857-41869

14. Ellis LM, Hicklin DJ: VEGF-targeted therapy: mechanisms of antitumour activity. Nat Rev Cancer 2008, 8:579-591

15. Ferrara N, Keyt B: Vascular endothelial growth factor: basic biology and clinical implications. EXS 1997, 79:209-232

16. Bergers G, Song S, Meyer-Morse N, Bergsland E, Hanahan D: Benefits of targeting both pericytes and endothelial cells in the tumor vasculature with kinase inhibitors. J Clin Invest 2003, 111:1287-1295

17. Dvorak HF: Tumors: wounds that do not heal-redux. Cancer Immunol Res 2015, 3:1-11

18. Jain RK: Antiangiogenesis strategies revisited: from starving tumors to alleviating hypoxia. Cancer Cell 2014, 26:605-622

19. Sitohy B, Nagy JA, Jaminet SC, Dvorak HF: Tumor-surrogate blood vessel subtypes exhibit differential susceptibility to anti-VEGF therapy. Cancer Res 2011, 71:7021-7028

20. Sitohy B, Nagy JA, Dvorak HF: Anti-VEGF/VEGFR therapy for cancer: reassessing the target. Cancer Res 2012, 72:1909-1914

21. Ke Q, Bodyak N, Rigor DL, Hurst NW, Chaplin DJ, Kang PM: Pharmacological inhibition of the hypertensive response to combretastatin A-4 phosphate in rats. Vascul Pharmacol 2009, 51: $337-343$

22. Liang WC, Wu X, Peale FV, Lee CV, Meng YG, Gutierrez J, Fu L, Malik AK, Gerber HP, Ferrara N, Fuh G: Cross-species vascular endothelial growth factor (VEGF)-blocking antibodies completely inhibit the growth of human tumor xenografts and measure the contribution of stromal VEGF. J Biol Chem 2006, 281:951-961

23. Camp ER, Yang A, Liu W, Fan F, Somcio R, Hicklin DJ, Ellis LM: Roles of nitric oxide synthase inhibition and vascular endothelial 
growth factor receptor-2 inhibition on vascular morphology and function in an in vivo model of pancreatic cancer. Clin Cancer Res 2006, 12:2628-2633

24. Wu Y, Zhong Z, Huber J, Bassi R, Finnerty B, Corcoran E, Li H, Navarro E, Balderes P, Jimenez X, Koo H, Mangalampalli VR, Ludwig DL, Tonra JR, Hicklin DJ: Anti-vascular endothelial growth factor receptor-1 antagonist antibody as a therapeutic agent for cancer. Clin Cancer Res 2006, 12:6573-6584

25. Wada Y, Li D, Merley A, Zukauskas A, Aird WC, Dvorak HF, Shih SC: A multi-gene transcriptional profiling approach to the discovery of cell signature markers. Cytotechnology 2011, 63:25-33

26. Chang SH, Kanasaki K, Gocheva V, Blum G, Harper J, Moses MA, Shih SC, Nagy JA, Joyce J, Bogyo M, Kalluri R, Dvorak HF: VEGF$\mathrm{A}$ induces angiogenesis by perturbing the cathepsin-cysteine protease inhibitor balance in venules, causing basement membrane degradation and mother vessel formation. Cancer Res 2009, 69:4537-4544

27. Holash J, Davis S, Papadopoulos N, Croll SD, Ho L, Russell M, Boland P, Leidich R, Hylton D, Burova E, Ioffe E, Huang T, Radziejewski C, Bailey K, Fandl JP, Daly T, Wiegand SJ, Yancopoulos GD, Rudge JS: VEGF-Trap: a VEGF blocker with potent antitumor effects. Proc Natl Acad Sci U S A 2002, 99:11393-11398

28. Tozer GM, Kanthou C, Baguley BC: Disrupting tumour blood vessels. Nat Rev Cancer 2005, 5:423-435

29. Facemire CS, Nixon AB, Griffiths R, Hurwitz H, Coffman TM: Vascular endothelial growth factor receptor 2 controls blood pressure by regulating nitric oxide synthase expression. Hypertension 2009, $54: 652-658$

30. Hood JD, Meininger CJ, Ziche M, Granger HJ: VEGF upregulates ecNOS message, protein, and NO production in human endothelial cells. Am J Physiol 1998, 274:H1054-H1058

31. Fukumura D, Kashiwagi S, Jain RK: The role of nitric oxide in tumour progression. Nat Rev Cancer 2006, 6:521-534

32. Lim KH, Ancrile BB, Kashatus DF, Counter CM: Tumour maintenance is mediated by eNOS. Nature 2008, 452:646-649

33. Tozer GM, Prise VE, Chaplin DJ: Inhibition of nitric oxide synthase induces a selective reduction in tumor blood flow that is reversible with L-arginine. Cancer Res 1997, 57:948-955

34. Murohara T, Horowitz JR, Silver M, Tsurumi Y, Chen D, Sullivan A, Isner JM: Vascular endothelial growth factor/vascular permeability factor enhances vascular permeability via nitric oxide and prostacyclin. Circulation 1998, 97:99-107

35. Ewer MS, Suter TM, Lenihan DJ, Niculescu L, Breazna A, Demetri GD, Motzer RJ: Cardiovascular events among 1090 cancer patients treated with sunitinib, interferon, or placebo: a comprehensive adjudicated database analysis demonstrating clinically meaningful reversibility of cardiac events. Eur J Cancer 2014, 50: $2162-2170$

36. Herrmann J, Yang EH, Iliescu CA, Cilingiroglu M, Charitakis K, Hakeem A, Toutouzas K, Leesar MA, Grines CL, Marmagkiolis K: Vascular toxicities of cancer therapies: the old and the new-an evolving avenue. Circulation 2016, 133:1272-1289

37. Maitland ML, Bakris GL, Black HR, Chen HX, Durand JB, Elliott WJ, Ivy SP, Leier CV, Lindenfeld J, Liu G, Remick SC, Steingart R, Tang WH; Cardiovascular Toxicities Panel, Convened by the Angiogenesis Task Force of the National Cancer Institute Investigational Drug Steering Committee: Initial assessment, surveillance, and management of blood pressure in patients receiving vascular endothelial growth factor signaling pathway inhibitors. J Natl Cancer Inst 2010, 102:596-604

38. Rini BI, Cohen DP, Lu DR, Chen I, Hariharan S, Gore ME, Figlin RA, Baum MS, Motzer RJ: Hypertension as a biomarker of efficacy in patients with metastatic renal cell carcinoma treated with sunitinib. J Natl Cancer Inst 2011, 103:763-773

39. Mourad JJ, Levy BI: Mechanisms of antiangiogenic-induced arterial hypertension. Curr Hypertens Rep 2011, 13:289-293

40. van der Veldt AA, de Boer MP, Boven E, Eringa EC, van den Eertwegh AJ, van Hinsbergh VW, Smulders YM, Serne EH: Reduction in skin microvascular density and changes in vessel morphology in patients treated with sunitinib. Anticancer Drugs 2010, 21:439-446

41. Inai $\mathrm{T}$, Mancuso $\mathrm{M}$, Hashizume $\mathrm{H}$, Baffert F, Haskell A, Baluk $\mathrm{P}$, Hu-Lowe DD, Shalinsky DR, Thurston G, Yancopoulos GD, McDonald DM: Inhibition of vascular endothelial growth factor (VEGF) signaling in cancer causes loss of endothelial fenestrations, regression of tumor vessels, and appearance of basement membrane ghosts. Am J Pathol 2004, 165:35-52

42. Su J, Laursen BE, Eskildsen-Helmond Y, Horsman MR, Simonsen U: The vascular-disrupting agent, combretastatin-A4-phosphate, enhances neurogenic vasoconstriction in rat small arteries. Eur J Pharmacol 2012, 695:104-111

43. Goffin JR, Straume O, Chappuis PO, Brunet JS, Begin LR, Hamel N, Wong N, Akslen LA, Foulkes WD: Glomeruloid microvascular proliferation is associated with p53 expression, germline BRCA1 mutations and an adverse outcome following breast cancer. $\mathrm{Br} \mathrm{J}$ Cancer 2003, 89:1031-1034

44. Straume O, Chappuis PO, Salvesen HB, Halvorsen OJ, Haukaas SA, Goffin JR, Begin LR, Foulkes WD, Akslen LA: Prognostic importance of glomeruloid microvascular proliferation indicates an aggressive angiogenic phenotype in human cancers. Cancer Res 2002, 62:6808-6811

45. Brat DJ, Van Meir EG: Glomeruloid microvascular proliferation orchestrated by VPF/VEGF: a new world of angiogenesis research. Am J Pathol 2001, 158:789-796

46. Dome B, Timar J, Paku S: A novel concept of glomeruloid body formation in experimental cerebral metastases. J Neuropathol Exp Neurol 2003, 62:655-661

47. Weidner N, Folkman J: Tumoral vascularity as a prognostic factor in cancer. Important Adv Oncol 1996:167-190

48. Udan RS, Vadakkan TJ, Dickinson ME: Dynamic responses of endothelial cells to changes in blood flow during vascular remodeling of the mouse yolk sac. Development 2013, 140:4041-4050

49. Huang Y, Goel S, Duda DG, Fukumura D, Jain RK: Vascular normalization as an emerging strategy to enhance cancer immunotherapy. Cancer Res 2013, 73:2943-2948

50. Arjaans M, Schroder CP, Oosting SF, Dafni U, Kleibeuker JE, de Vries EG: VEGF pathway targeting agents, vessel normalization and tumor drug uptake: from bench to bedside. Oncotarget 2016, 7: 21247-21258 